\title{
SYMPATHECTOMY AND THE PALLIATIVE OPERATIONS FOR OCCLUSIVE ARTERIAL DISEASE
}

\author{
By A. J. SLessor, M.V.O., F.R.C.S. \\ From the Department of Surgery, University of Edinburgh
}

\section{Sympathectomy in Occlusive Arterial Disease}

Sympathectomy may be indicated in arteriosclerosis obliterans and is the treatment of choice in thromboangiitis obliterans, but it is imperative that the disease process has not advanced so far as to make the operation ineffectual.

\section{Arteriosclerosis}

After due attention has been paid to the reduction of weight in the obese, the control of diabetes, the correction of anaemia, the necessity of avoiding even trivial injury or exposure to cold, the wearing of suitable clothing and abstinence from smoking, consideration of the following points enables the surgeon to decide whether or not sympathectomy should be performed.

I. Symptomatology. The commonest symptom is intermittent claudication in the muscles of the calf. Because dilatation of the vessels in muscle during activity depends on metabolites produced by muscle action and not on alteration in vasomotor tone, sympathectomy cannot by direct effect on the muscle vessels either abolish or lessen claudication pain. If the arterial block is at a high level, however, as in the iliac or upper femoral arteries, sympathectomy may relieve claudication by increasing the collateral circulation which bypasses the thrombosed segment and so making available a greater amount of blood to meet the needs of the calf during exercise; the level of arterial block is established by clinical examination of the pulses and may be confirmed by oscillometry and arteriography. Occasionally patients are seen who suffer from claudication in the calf and yet have all pulses palpable in the limb; such a finding indicates either that the vessels which supply the calf muscles are themselves thrombosed or that during exercise sufficient spasm occurs in the large vessels to reduce the blood supply of the muscles to claudication level. Examination of the pulses, immediately after exercise sufficient to cause claudication, suffices to separate the two conditions; if the distal pulses persist, sympathectomy is valueless; if the distal pulses disappear, sympathectomy may be performed with confidence, especially since these patients will sooner or later produce signs of vessel obliteration. Sympathectomy will also lessen claudication if the latter is accompanied by a feeling that the foot becomes cool or cold during exercise.

Because the main object of sympathectomy is the improvement of peripheral nutrition it should be performed when the main symptom is coldness of the feet, paraesthesiae, rest pain or neuritic pain due to ischaemia, provided always the reflex vasodilatation test described below is satisfactory. The operation will be indicated, therefore, even when the presenting symptom is claudication, if there are objective signs of failing peripheral nutrition; the relief of claudication will depend on the level of arterial block, as already indicated, but sympathectomy will offer the best insurance against gangrene; unfortunately it cannot be offered as aguarantee.

2. Response to Special Investigations. While there are numerous ways of inducing vasodilatation in a limb, the author finds the reflex method the greatest help in deciding for or against sympathectomy. The lower extremities of the patient are exposed from the knees down in a room the temperature of which is maintained at about $20^{\circ} \mathrm{C}$. by thermostatic control; all draughts must be excluded. By copper-constantan thermocouple and galvanometer the temperature of the digits is recorded for an observation period of 20 minutes; heat is then applied elsewhere to the body as by immersing an upper extremity in water at $44^{\circ} \mathrm{C}$., or by applying an electric blanket to the trunk or an electric pad and rubber hot water bottle to an upper limb. Recordings of the digital skin temperature are made every five minutes. In the healthy the temperature of the toe starts to rise from its resting level of about $22^{\circ} \mathrm{C}$. in approximately ${ }_{5} 5$ minutes and progresses in a short time to full vasodilatation (about $35^{\circ} \mathrm{C}$.). When there is severe occlusive arterial disease this reflex vasodilatation does not occur; in moderately severe arterial disease some vasodilatation will occur, although the onset may be delayed and the rise in temperature less steep. Arterial spasm, which is frequently associated with arteriosclerosis, 
will gradually be abolished during the examination and reflex vasodilatation will occur. If the limb warms to over $30^{\circ} \mathrm{C}$. sympathectomy is firmly indicated, but even a small rise in temperature indicates that the limb is capable of some vasodilatation and the author advises sympathectomy if the limb warms through $3^{\circ} \mathrm{C}$. The immediate result after operation is frequently much more satisfactory than that expected in such cases, and it is certain that in the past a number of patients have been denied the benefits of sympathectomy. It is true, however, that the quicker and higher the skin temperature rises the better and more permanent will be the insurance against gangrene.

In patients with severe ischaemia it is advisable to expose only the feet and to reduce the period of observation to ro minutes. When severe organic disease is associated with spasm induced by exposure, reflex vasodilatation may not occur and a deserving patient may be refused operation.

The author does not hold that the reflex vasodilatation test is a more accurate method than lumbar sympathetic block by novocain or nerve block or spinal anaesthesia; it seems to him, however, that it is more valuable in indicating not only what the immediate effect of operation will be but also what the more lasting effect will be.

The estimation of blood flow through a digit or the forefoot may be used to determine the extent to which peripheral blood supply will be altered by sympathectomy. Meaning as it does actual flow in terms of millilitres of blood per $100 \mathrm{cc}$. of tissue per minute, this method is held by some to be more accurate than skin temperature estimation.

3. Age. At one time arteriosclerosis was regarded as an absolute contraindication to sympathectomy. Later patients up to the age of $5^{\circ}$ years were accepted, then the age limit was raised to 60 years. Now it may be said that, within reason, patients of any age may be subjected to operation, provided all other criteria are observed.

4. Generalized Arteriosclerosis. Evidence of coronary or cerebral or renal involvement contraindicates operation. A history of angina pectoris means that not only sympathectomy but also the palliative operations must be refused. Unfortunately a large number of patients whose arteriosclerosis first affects their peripheral vessels subsequently succumb to coronary disease.

5. The Time of Operation. When there is marked nutritional failure at the periphery, as is indicated by persistent severe rest pain, it is of advantage to delay operation for a period. The patient should be kept in bed, the head of which is raised on 6-in. blocks, the feet should be exposed under a cage to room temperature and gradual reflex vasodilatation should be induced. Only when some improvement in nutrition occurs should sympathectomy be considered; if the operation is performed too early in the presence of severe peripheral ischaemia, whether there already is gangrene or not, gangrene may be precipitated or caused to extend by the occurrence of oedema at the actual or potential demarcation zone, where dead or near-dead tissue adjoins just-living tissue.

\section{Thromboangiitis Obliterans}

In this condition sympathectomy is the treatment of choice. 'The deleterious effect of smoking is so definite, however, that complete cessation is imperative.

Provided that reflex vasodilatation occurs, operation should be undertaken. As in arteriosclerosis, if there is severe peripheral ischaemia a period of rest in bed with gradual reflex vasodilatation is beneficial.

\section{Lumbar Sympathectomy}

The patient is placed in the oblique position, the trunk making an angle of $45^{\circ}$ with the table in the coronal plane. The incision starts just below the tip of the 12 th rib and is extended forwards towards the umbilicus for about $10 \mathrm{~cm}$. The three layers of muscle are divided in the same line. The peritoneum is mobilized forwards and medially, the finger entering between peritoneurg and iliac fascia. Blunt dissection is continue inwards, exposing the psoas under the iliac fascio and the genitofemoral nerve which lies on the muscle. The ureter and testicular or ovarias vessels are carried forwards with the peritoneum and the vertebral attachment of psoas is exposed. The sympathetic chain lies on the vertebral bodies, being slightly overlapped by the aorta on the left side and appreciably overlapped by the inferior vena cava on the right side; these vessels require to be retracted medially with care, and for the atheromatous aorta the assistant's hand is less liable to produce thrombosis than is a metal retractor. The peritoneum is mobilized along this line upwards until the diaphragmatic crus is easily visualized and downwards until the common iliac vessel is seen. The chain is picked up by blunt hook and as the ganglia are exposed their rami are $\delta$ divided; danger to lumbar veins is avoided if they pass anterior to the chain, which they more com- 은 monly do on the right side, by dividing the chain $\rightarrow$ above or below the vein and pulling the segment through behind the vein. Care in lifting the chain forwards before the division of rami suffices to 0 ensure the safety of lumbar veins passing behind $N$ the chain. Dissection is usually begun below, at $\mathrm{N}_{\mathrm{J}}$ the third lumbar ganglion and continued upwards; 0 in order to extirpate the first ganglion it is necessary to divide the fascial or muscular prolongation of $\underset{D}{\sim}$

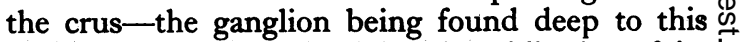
structure and recognized by the obliquity of its 0 rami. The chain should be excised from above the $\vec{D}$ 
first ganglion to below the third; if the lower ganglia are inconstant in position or if they are absent it is recommended that in all $10 \mathrm{~cm}$. of chain be removed. The muscles are closed in layers by continuous catgut sutures and the skin by fine continuous catgut supplemented by an anchor dressing.

When the better leg also shows signs of ischaemia it may be sympathectomized by turning the patient round and repeating the procedure on that side. The bilateral operation is tolerated as well as is the unilateral operation. It is wrong, however, to perform the bilateral operation routinely; operation should be performed only if the second leg already is ischaemic, since its value in increasing collateral circulation will be greater when arterial occlusion is present.

On properly selected patients the mortality from lumbar sympathectomy is insignificant.

There are three side-effects: (I) There is anhidrosis in the sympathectomized foot; the rubbing in of olive oil prevents the skin from becoming too hard and scaly. (2) In the male, bilateral extirpation of the first lumbar ganglion may lead to sterility, although this is not invariable. (3) In the older age groups there may develop an annoying pain in the thigh; it occurs some seven days after operation and is described as a gnawing deep-seated pain, usually worse at night; the skin over the antero-lateral aspect of the thigh may be hyperaesthetic so that the touch of bedclothes is painful. The cause of the pain is not understood. As a rule relief occurs in two to three weeks, so that there is no reason 'for withholding the simpler analgesics; on occasion the pain may last some three months but it is invariably self-terminating.

\section{The Results of Sympathectomy}

1. Arteriosclerosis. In the properly selected patient who is willing to cooperate, sympathectomy can do much to relieve symptoms and is the only method of achieving local vasodilatation where it is most needed. It has to be kept in mind, however, that arteriosclerosis is a progressive disease and that while sympathectomy offers the best insurance against gangrene, etc., there can be no guarantee.

2. Thromboangiitis Obliterans. This condition also tends to be progressive and is almost invariably so if the patient does not stop smoking. In a number of cases, however, the disease appears to burn itself out and sympathectomy may tide the patient over the most acute phases and prevent tissue loss. When the condition progresses it can be claimed not only that sympathectomy delays the onset of gangrene but also that it may permit a below-knee amputation to be performed, a great asset to a patient for whose second leg the outlook is insecure.

\section{Palliative Operations in Occlusive Arterial Disease}

The object of palliative operations is to increase claudication distance. Palliative operations have no effect on peripheral nutrition, although some patients state that their foot feels warmer after operation. The operations should be performed, therefore, only when the nutrition of the toes and foot is satisfactory with, at most, only minimal signs of nutritional upset; they are of value in those patients whose claudication is due to occlusion of the arteries to the calf muscles themselves (see above) and they may be performed after sympathectomy has improved peripheral nutrition without relieving claudication of disabling severity.

I. Myoneurectomy. Muscle which is inactive does not require an increased blood supply during exercise, metabolites do not accumulate and therefore claudication does not occur. Gastrocnemius and the upper two-thirds of soleus may be denervated through an incision $7 \mathrm{~cm}$. in length situated over the lower part of the popliteal fossa; the branches to the two heads of gastrocnemius and the upper branch to soleus are resected for a length of $3 \mathrm{~cm}$.

Patients are encouraged to walk on the day after operation and it is advisable to administer anticoagulants and to support the limb by crepe bandage for a few days in order to lessen the risk of thrombosis which may result from the stagnation of blood in the veins of the paralyzed muscle.

The operation allows the patient to walk on the average about five times the pre-operative claudication distance. There is inevitably a loss in the spring of the limb while walking but this is of little consequence to a patient who has had severe claudication.

2. Tenotomy of the Tendo Achilles. In some centres this operation has replaced myoneurectomy. The tendon is divided by tenotomy at the heel, just high enough to avoid pressure by the shoe on the scar; it requires only brief anaesthesia under pentothal and is bloodless. Patients are as a rule able to walk at least their pre-operative claudication distance on the following day and although some complain of local discomfort at the site of tendon section they do not suffer claudication.

If the second leg requires operation the second tenotomy is best delayed for about three months, by which time full stability has been regained in the first limb, the tendon being reconstituted in length by fibrous tissue.

As occurs after myoneurectomy, there is a loss of spring in the gait but the ease with which considerable distances can in time be covered at reasonable speed is gratifying to both patient and surgeon. 